\title{
Final report for the NSF/DOE partnership in basic plasma science grant DE-FG02-06ER5406 'Laser-driven collisionless shocks in the Large Plasma Device'
}

\author{
C. Niemann ${ }^{1}(\mathrm{PI})$ \\ Collaborators: \\ W. Gekelman ${ }^{1}$, D. Winske ${ }^{2}$, D. Larson ${ }^{3}$ \\ ${ }^{1}$ University of California Los Angeles, 1040 Veteran Ave., Los Angeles, CA 90095 \\ ${ }^{2}$ Los Alamos National Laboratory \\ ${ }^{3}$ Lawrence Livermore National Laboratory
}

October 2012

\section{Summary}

We have performed several thousand high-energy laser shots in the LAPD to investigate the dynamics of an exploding laser-produced plasma in a large ambient magneto-plasma. Debris-ions expanding at super-Alfvenic velocity (up to $\mathrm{M}_{\mathrm{A}}=1.5$ ) expel the ambient magnetic field, creating a large $(>20 \mathrm{~cm})$ diamagnetic cavity. We observed field compressions of up to $\mathrm{B} / \mathrm{B}_{0}=1.5$ at the edge of the bubble, consistent with the MHD jump conditions, as well as localized electron heating at the edge of the bubble. Two-dimensional hybrid simulations reproduce these measurements well and show that the majority of the ambient ions are energized by the magnetic piston to super-Alfvenic speeds and swept outside the bubble volume. Nonlinear shear-Alfven waves $\left(\delta \mathrm{B} / \mathrm{B}_{0}>25 \%\right)$ are radiated from the cavity with a coupling efficiency of $70 \%$ from magnetic energy in the bubble to the wave. While the data is consistent with a weak magnetosonic shock, the experiments were severely limited by the low ambient plasma densities $\left(10^{12} \mathrm{~cm}^{-3}\right) .2 \mathrm{D}$ hybrid simulations indicate that future experiments with the new LAPD plasma source and densities in excess of $10^{13} \mathrm{~cm}^{-3}$ will drive full-blown collisionless shocks with $\mathrm{M}_{\mathrm{A}}>10$ over several $\mathrm{c} / \mathrm{w}_{\mathrm{pi}}$ and shocked Larmor radii. In a separate experiment at the LANL Trident laser facility we have performed a proof-of-principle experiment at higher densities to demonstrate key elements of collisionless shocks in laser-produced magnetized plasmas with important implications to NIF. Simultaneously we have upgraded the UCLA glass-laser system by adding two large amplitude disk amplifiers from the NOVA laser and boost the on-target energy from 30 $\mathrm{J}$ to up to $1 \mathrm{~kJ}$, making this one of the world's largest university-scale laser systems. We now have the infrastructure in place to perform novel and unique high-impact experiments on collisionless shocks at the LAPD.

\section{Collisionless shock experiments at the LAPD}

\section{I.1. EXPERIMENTAL SETUP}

The experiments were performed with the Phoenix Nd:glass-laser system and the LAPD. The LAPD creates highly magnetized plasmas that are large enough (18 m length, $60 \mathrm{~cm}$ diameter) to support Alfven waves. Plasmas are typically produced at densities up to $\mathrm{n}_{\mathrm{i}}=2 \times 10^{12} \mathrm{~cm}^{-3}$, electron temperatures around $\mathrm{T}_{\mathrm{e}}=5 \mathrm{eV}$, and ion temperatures around $\mathrm{T}_{\mathrm{i}}=1 \mathrm{eV}$. The quiescent and currentfree plasma is created in helium or neon in a steady, axial magnetic field of 275-1800 G by 
pulsing a barium oxide coated $(\mathrm{BaO}-\mathrm{Ni})$ cathode at one end of the machine negatively with respect to an anode grid $30 \mathrm{~cm}$ away. A few $\mathrm{ms}$ after the discharge is initiated, the plasma reaches a steady state, and a 0.5 " thick graphite-target embedded in the preformed plasma is irradiated with a laser energy up to $25 \mathrm{~J}$.

The high-energy laser system consists of a Q-switched Nd:YAG oscillator (1064 nm, $10 \mathrm{~mJ}, 5 \mathrm{~ns}$ FWHM), $7 \mathrm{~mm} \mathrm{Nd:YAG} \mathrm{pre-amplifier,} \mathrm{and} \mathrm{four} \mathrm{flashlamp-pumped} \mathrm{Nd:silicate} \mathrm{rod-amplifiers}$ ( $25 \mathrm{~mm}$ double-pass, $32 \mathrm{~mm}$, and $45 \mathrm{~mm}$ ) that boost the $10 \mathrm{~mJ}$ seed-pulse to an out- put energy of $30 \mathrm{~J}$ (25 J in the P-polarized component). A $30 \mathrm{~m}$ long beamline in air transports the $40 \mathrm{~mm}$ diameter beam from the laser-laboratory one story down to the LAPD. A combination of quarterwaveplate and thin-film polarizers is used to optically isolate the laser- system from the target, preventing laser damage due to backscatter and creating a circularly polarized beam on target. An aspheric $\mathrm{f} / 6$ doublet-lens inside the plasma vessel creates a tight focus $(50 \mu \mathrm{m}$ FWHM) and peak intensities up to $10^{13} \mathrm{~W} / \mathrm{cm}^{2}$ at $20 \mathrm{~J}$ with an energy stability of $10 \%$. The on-target intensity could be changed during the experiments by translating the focusing lens closer to the target by several $\mathrm{cm}$. The target normal was aligned exactly perpendicular to the external field to direct the blowoff plume into a transverse direction. The flat target surface was offset $13 \mathrm{~cm}$ from the axis of the LAPD, providing roughly $40 \mathrm{~cm}$ of ambient plasma to interact with as the laser-plasma expands perpendicularly across the magnetic field (Fig. 1). After each shot, the target was translated by a few $\mathrm{mm}$ to provide a fresh surface. In the following, all data will be expressed in LAPDcoordinates (see Fig. 1), where the external B-field points in the positive z-direction, the laser blow-off propagates horizontally in negative $\mathrm{x}$-direction, and the transverse field of the shearwaves is typically measured where it is vertical (y-direction). Relative distances from the target along these coordinates will be denoted as $\Delta \mathrm{x}$, and $\Delta \mathrm{y}$ in transverse direction, and $\Delta \mathrm{z}$ axially.

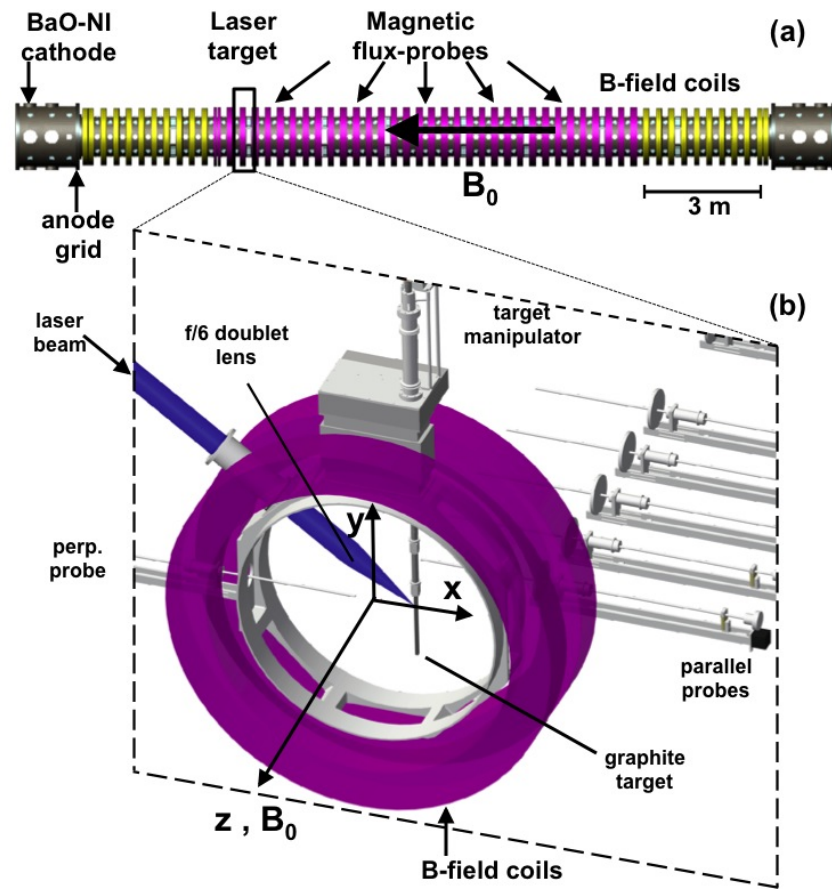

FIG. 1 Experimental setup at the LAPD (a) and details of the laser-target configuration (b). 
An array of ten magnetic flux probes (i.e. Bdot probes) was distributed along the machine at distances $\Delta \mathrm{z}$ up to $7 \mathrm{~m}$ from the target to measure the magnetohydrodynamic (MHD) response of the ambient plasma along the direction of the magnetic field. One additional probe was inserted from a port opposing the target to investigate the dynamics of the diamagnetic cavity perpendicular to the external field. All probes could be positioned at arbitrary transverse positions (x-axis) using motorized probe- drives with sub-mm precision. The reproducibility of the ambient plasma, along with its $1 \mathrm{~Hz}$ repetition rate and the high-shot rate of the laser system (5-10 shots/hour) allowed detailed scans of the magnetic field over large spatial regions in the plasma. The three-dimensional magnetic pickup coils were differentially wound on a $1 \mathrm{~mm}$ or $3 \mathrm{~mm}$ cube core (10 turns each axis) and were calibrated to frequencies of up to $100 \mathrm{MHz}$. Probe signals were recorded using custom-built $100 \mathrm{MHz}$ differential amplifiers coupled to $14 \mathrm{bit}, 100 \mathrm{MS} / \mathrm{s}$ digitizers, and numerically integrated to compensate for the individual frequency response. In addition, plasma density and ion time-of-flight measurements were performed with double sided Langmuir probes.

\section{I.2 Evolution of the diamagnetic cavity}

The experiments were performed in a perpendicular geometry (i.e. target-normal and laser blowoff direction aligned perpendicular to the external field) to investigate the formation and collapse of the diamagnetic cavity in the presence of the ambient magnetized plasma. The debris ions have directed Larmor radii of several tens of $\mathrm{cm}$ and are essentially unmagnetized. The lighter electrons are tied to the field lines $\left(\mathrm{r}_{\text {Larmor,e }}<1 \mathrm{~mm}\right)$, causing a radial electric field and azimuthal electron $\mathrm{E} \times \mathrm{B}$ drift. These currents reinforce the pressure driven diamagnetic electron-drift due to plasma density gradients at the leading-edge of the exploding plasma $(\nabla \mathrm{P} \times \mathrm{B})$ and thus create azimuthal currents that ultimately expel the external field. The azimuthal electric field also accelerates ambient ions that start to $\mathrm{E} \times \mathrm{B}$-drift radially in a quarter gyroperiod (i.e. Larmor coupling).

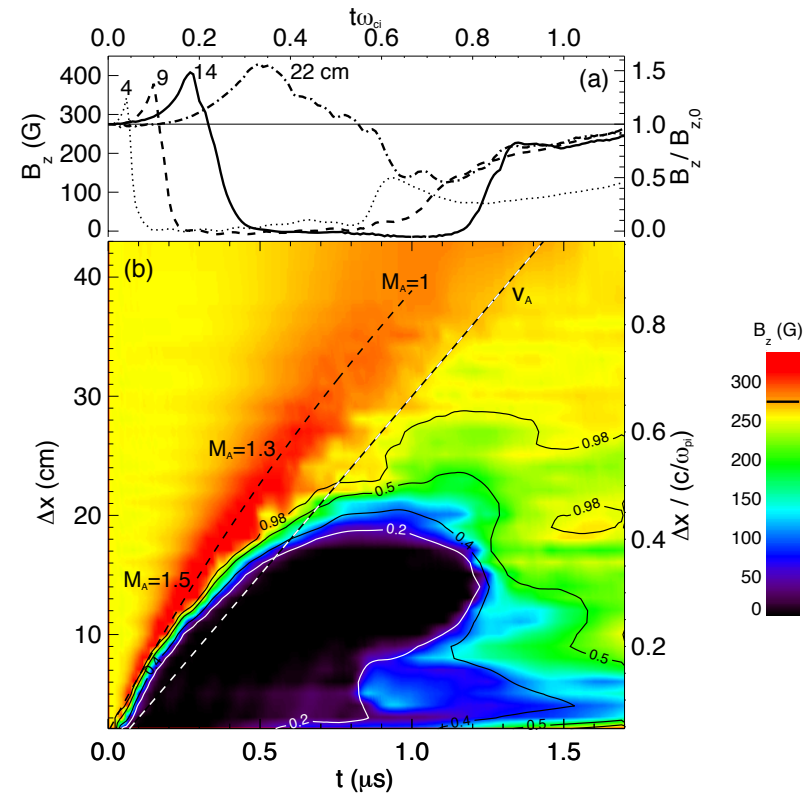

Fig. 2. Magnetic-flux probe measurements of the evolution of the magnetic field $(\mathrm{Bz})$ at various distances from the target in $\mathrm{He}+$ at $275 G$ (a). The field compression increases with distance from the target, reaching a steady $B / B_{0}=1.5$ from 14 to $22 \mathrm{~cm}$. Inside the bubble the field is fully expelled but diffuses back into the bubble-volume after about $1 \mu$ s. (b) The contour-plot of the same data is a composite of 80 laser shots and shows Bz as a function of time with a spatial resolution of $5 \mathrm{~mm}$.

Figure 2 shows magnetic probe measurements of the formation and collapse of the diamagnetic cavity that is formed by a laser-produced carbon-plume exploding into a stationary $\mathrm{He}^{+}$plasma at upstream (i.e. the unperturbed region ahead of the piston) conditions of $n_{i}=9 \times 10^{11} \mathrm{~cm}^{-3}$ and $T_{e}=5$ $\mathrm{eV}$ in $275 \mathrm{G}$. The complete expulsion of the field and compression into a thin shell as well as the 
overall size and lifetime of the cavity are clearly visible in the contour plot (Fig. 2b), which combines single-point measurements from 80 separate laser shots conducted over the course of two days. To obtain this dataset one magnetic field probe was moved to a different transverse position (x-axis) for each shot, while the experiment was repeated at reproducible laser-target and ambient plasma parameters. The magnitudes of the transverse magnetic field components $\left(\mathrm{B}_{\mathrm{x}}\right.$ and $\mathrm{B}_{\mathrm{y}}$, not shown) are much smaller at a few Gauss since measurements were performed only at points along the blow-off direction and perpendicular to the initial field, where the expelled and compressed field is mostly parallel to the direction of the external magnetic field.

The magnetic pulse expands initially at $\mathrm{v}_{\text {piston }}=5 \times 10^{7} \mathrm{~cm} / \mathrm{s}$ and is super-Alfvenic at $\mathrm{M}_{\mathrm{A}}=\mathrm{v}_{\text {piston }} / \mathrm{v}_{\mathrm{A}}=1.5$. The pulse is also hypersonic at $\mathrm{M}_{\mathrm{s}}=\mathrm{v}_{\text {piston }} / \mathrm{v}_{\mathrm{s}}=35$, where $\mathrm{v}_{\mathrm{s}}$ is the ion-sound speed. This velocity is consistent with empirical scaling laws for the ablation plume at the laser parameters used. The field compression factor increases as the bubble grows and reaches a maximum value of $\mathrm{B} / \mathrm{B}_{0}=1.5$ at a distance of $14 \mathrm{~cm}$ from the target. This compression ratio is maintained until the bubble stagnates at $22 \mathrm{~cm}$ and is consistent with the Rankine-Hugoniot jump conditions for a shock, although the piston only travels for a fraction of a gyroperiod $\left(\mathrm{t} \omega_{\mathrm{ci}}=0.5\right.$ considering the initial ambient plasma parameters, or $t \omega_{\mathrm{ci}}=0.75$ in the downstream region considering field compression). The thickness of the pulse is around $1 \mathrm{~cm}$ and on the order of 10 $\mathrm{c} / \omega_{\mathrm{pe}}$, which is comparable to the thickness of the ramp in a collisionless shock. When the bubble stagnates and the piston stops, the magnetic compression continues to propagate as a fast magnetosonic pulse through the ambient plasma, gradually decreasing in speed to $\mathrm{M}_{\mathrm{A}}=1$ at twice the bubble stopping radius. This pulse eventually dissipates due to geometrical effects and collisional and electron Landau damping in the upstream plasma, and broadens to a width of several tens of electron-skin-depths $\left(\mathrm{c} / \omega_{\mathrm{pe}}\right)$. Hybrid simulations of the formation time of perpendicular shocks show that a shock can form in two ways, either by a hot ion-beam or by a cloud of hot electrons. In the hot-electron case, which is more relevant to the experiment described here, coupling between the debris-plasma and the ambient plasma is provided by the presence of laminar electric fields due to the electron pressure gradient across the bubble interface. The formation time is on the order of $\omega^{-1}$ depending on the electron $\beta$ (i.e. the ratio of plasma energy density to magnetic field energy density), and is quite close to the duration of our experiment. Shock formation is slower in the hot-ion case, where coupling is due to the Larmor motion of the energetic ions, as in most extraterrestrial shocks.

The maximum size of the bubble is around $23 \mathrm{~cm}$ when defined as the volume where the ambient field is reduced by more than half. Complete field expulsion occurs only in a volume with a size of around $17 \mathrm{~cm}$. We note, however, that some field reduction is observed out to distances in excess of $30 \mathrm{~cm}$. The size of a diamagnetic bubble can be estimated from a simple energy balance, considering that the kinetic energy of the debris $E_{\text {debris }}=V \cdot B^{2} /\left(2 \mu_{0}\right)+1 / 2 \cdot n_{i} m_{i} V v^{2}$ is fully converted to magnetic energy in the bubble volume $\mathrm{V}$ and kinetic energy of the ambient ions that start streaming in the bubble explosion direction due to Larmor coupling. Hybrid simulations show that Larmor-coupling accelerates the initially stationary ambient ions to the piston velocity in a quarter gyroperiod $\pi / 2 \cdot \omega_{\mathrm{ci}}$. For the present parameters the bubble stagnates after $\mathrm{t}=0.7 \mu \mathrm{s}$ or $\mathrm{t} \omega_{\mathrm{ci}} \approx 0.5$ and the ambient ions in at least half of the bubble volume should acquire the piston velocity. The magnetic energy contained in the bubble volume is around $1.3 \mathrm{~J}$, which is only $6 \%$ of the on-target laser energy. Considering that about half of the ambient ions expelled from the bubble should have acquired the piston velocity and a total energy around $2 \mathrm{~J}$, the overall conversion efficiency from the laser to the ambient medium would be around $13 \%$. We note, however, that there is no measurement of the ambient ion velocity in the present experiment and no in-situ comparison with a vacuum expansion to quantify the coupling to the background ions. Without Larmor-coupling (or laminar coupling) the energy now lost to kinetic energy of streaming ambient ions would be available for additional field expulsion and should increase the 
size of the bubble by $30 \%$. Experiments performed at identical laser-target parameters in a neon background $(\mathrm{A}=20)$ yield the same bubble-stopping radius within the accuracy of the measurement. While neon-ambient ions moving at an identical velocity as the helium ions would contain a factor of five more energy, Larmor coupling proceeds five times slower so that not much momentum transfer is expected for neon at the cur- rent conditions, consistent with the observed insensitivity of the bubble size to the ambient ion mass. The bubble size roughly obeys the $\left(E / B^{2}\right)^{1 / 3}$ scaling in experiments with varying magnetic field, and reduces to $11 \pm 2 \mathrm{~cm}$ and $5 \pm 2$ $\mathrm{cm}$ diameters for $600 \mathrm{G}$ and $1800 \mathrm{G}$, respectively. This indicates that coupling between the debris ions and the ambient plasma is comparable in all three cases. This assumption is reasonable, since higher fields will not only decrease the ion-gyroperiod $\left(\omega_{\mathrm{ci}}{ }^{-1}\right)$ but also the bubble size and thus the piston transit time. We observe variations in the peak compression $\mathrm{B} / \mathrm{B}_{0}$ at the cavity edge with $\mathrm{M}_{\mathrm{A}}$. Reducing the blow-off speed to $3.5 \times 10^{7} \mathrm{~cm} / \mathrm{s}$ by defocusing the laser beam $\left(\mathrm{M}_{\mathrm{A}}=1.1\right)$ decreases the peak compression to $\mathrm{B} / \mathrm{B}_{0}=1.3$. In neon $\left(\mathrm{M}_{\mathrm{A}}=3.4\right)$ we measure compression ratios of 1.4. These are well below the values predicted by the magnetohydrodynamic jump-conditions, since an ambient neon plasma is only weakly magnetized and Larmor coupling is inefficient during the short bubble transit times. At $1800 \mathrm{G}$ in helium $\left(\mathrm{M}_{\mathrm{A}}=0.36\right)$, the measured peak compression was $\Delta \mathrm{B}=300 \mathrm{G}$ or $\mathrm{B} / \mathrm{B}_{0}=1.2$.

The cavity collapses roughly $0.5 \mu$ s after it stagnates and much faster than the magnetic diffusion time $\mu_{0} \sigma \mathrm{R}_{\mathrm{B}}^{2} \approx 100 \mu \mathrm{s}$, where $\sigma=50 \pi 1 / 2 \varepsilon_{0}^{2}\left(\mathrm{kT}_{\mathrm{e}}\right)^{3 / 2} /\left({ } \mathrm{m}_{\mathrm{e}} \mathrm{e}^{2} \mathrm{Z} \cdot \ln \Lambda\right)$ is the plasma Spitzerconductivity, and $\mathrm{R}_{\mathrm{B}}$ is the magnetic bubble radius. Initially the diffusion proceeds anomalously fast until the field in the bubble is restored to around $\mathrm{B} / \mathrm{B}_{0}=80 \%$. The diffusion rate then slows to near classical. Fast anomalous diffusion is a common feature of experiments on interpenetrating plasma clouds. Similar bubble-lifetimes have also been reported from experiments at the LAPD with smaller lasers, driving smaller cavities at lower blow-off velocities. Since the bubbles here are an order of magnitude larger in size, comparable diffusion times indicate significantly larger diffusion coefficients (by a factor of 100), consistent with the faster piston speeds and higher cross-field currents. We note that at later times, during the collapse of the cavity, we observe variations in both transverse field components that are comparable to the ambient field. The apparent early diffusion near the target (at $\Delta \mathrm{x}=3-8 \mathrm{~cm}$ and $0.8 \mu \mathrm{s}$ in Fig. $2 \mathrm{~b}$ ) is in fact due to a rotation of the field direction from $\mathrm{B}_{\mathrm{z}}$ to $\mathrm{B}_{\mathrm{x}}$ and $\mathrm{B}_{\mathrm{y}}$, probably caused by geometrical effects near the edge of the bubble.

The magnetic field data displays high-frequency oscillations in the lower-hybrid range, indicative of the growth of micro-instabilities responsible for the anomalous field diffusion. Similar pulsations are observed in the ion-saturation current from Langmuir probes (see next section). The nature of these high-frequency oscillations is a topic under investigation. Magnetic fluctuations inside the cavity are also observed in hybrid simulations, especially near the edge of the bubble. The large-Larmor radius Rayleigh-Taylor instability grows to large amplitudes on time-scales faster than $\omega_{\mathrm{ci}}{ }^{-1}$ due to the population of fast debris ions with Larmor-radii larger than the bubble stopping radius. Also the modified two-stream instability and the lower-hybrid drift instability have been proposed or observed to explain fast field diffusion in earlier work. Experiments performed with identical ambient plasma parameters but increased focal spot size and thus lower blow-off velocity $\left(3.5 \times 10^{7} \mathrm{~cm} / \mathrm{s}\right)$ display similar oscillations inside the cavity but at significantly reduced amplitude (factor 2-5). Simultaneously, the diffusion time increases from $0.5 \mu \mathrm{s}$ to $2 \mu \mathrm{s}$. This behavior is consistent with a reduced growth of micro-instabilities when the cross-field current at the edge of the bubble decreases.

\section{I.3 Fast electron generation at the bubble edge}

Figure 3 shows ion-saturation-current measurements inside the diamagnetic cavity performed with a Langmuir probe biased at a fixed potential of $-70 \mathrm{~V}$ with respect to the anode of the discharge circuit, which is at plasma potential. The probe thus excludes all bulk plasma electrons 
and measures both ion saturation current $\left(\mathrm{I}_{\text {sat }}\right)$ and electrons with sufficient energy to overcome the $70 \mathrm{~V}$ probe bias, corresponding to velocities above $3.5 \times 10^{8} \mathrm{~cm} / \mathrm{s}$. The exposed area of the probe was positioned such that it was facing away from the laser target. The voltage drop across a $10 \Omega$ resistor was measured through an optical isolator on a $100 \mathrm{MS} / \mathrm{s}, 14$ bit digitizer. The contour plot (Fig. 3a) shows a composite of 20 laser shots where the probe was moved transverse to the external magnetic field in 1 or $2 \mathrm{~cm}$ increments as the experiment was repeated with reproducible laser and plasma parameters identical to those in Fig. 2. We observe a negative double-spike at the edge of the bubble out to distances of $15 \mathrm{~cm}$ from the target due to electrons above $70 \mathrm{eV}$. Inside the bubble we observe the positive ion- saturation current from the laserblow-off plasma. The first negative spike coincides with the ramp on the Bdot-probe signal where the magnetic field is compressed (Fig. $3 \mathrm{~b})$ and is due to adiabatic heating $(\mathrm{dB} / \mathrm{dt}>0)$. The second negative spike occurs at the time of maximum field expulsion and may be due to Ohmic heating by the diamagnetic current, or by anomalous heating by micro-instabilities. Inside the bubble, the plasma density $\mathrm{n}$ can be calculated from the ion saturation current $\mathrm{I}_{\mathrm{sat}}=0.61 \mathrm{neA}\left(\mathrm{kT}_{\mathrm{e}} / \mathrm{m}_{\mathrm{i}}\right)^{1 / 2}$, if the temperature $T_{e}$ is known $\left(A=1 \mathrm{~mm}^{2}\right.$ is the exposed area of the electrode, and $m_{i}$ is the ion mass). A temperature around $10 \mathrm{eV}$ several $\mathrm{cm}$ from the laser target was measured in a different experiment with similar parameters, in good agreement with radiation-hydrodynamic simulations. The measured ion-saturation current is roughly consistent with these temperatures and $10^{15}$ debris ions ablated from the target, assuming that the bulk of these ions are stopped and evenly distributed throughout the bubble as suggested by the data. The $\mathrm{I}_{\text {sat }}$ signal inside the bubble is approximately constant over $2 \mu \mathrm{s}$, even though the bubble collapses at around $\mathrm{t}=1 \mu \mathrm{s}$. After $2 \mu \mathrm{s}$ the plasma dissipates, and the probe no longer draws a detectable ion-saturation current.

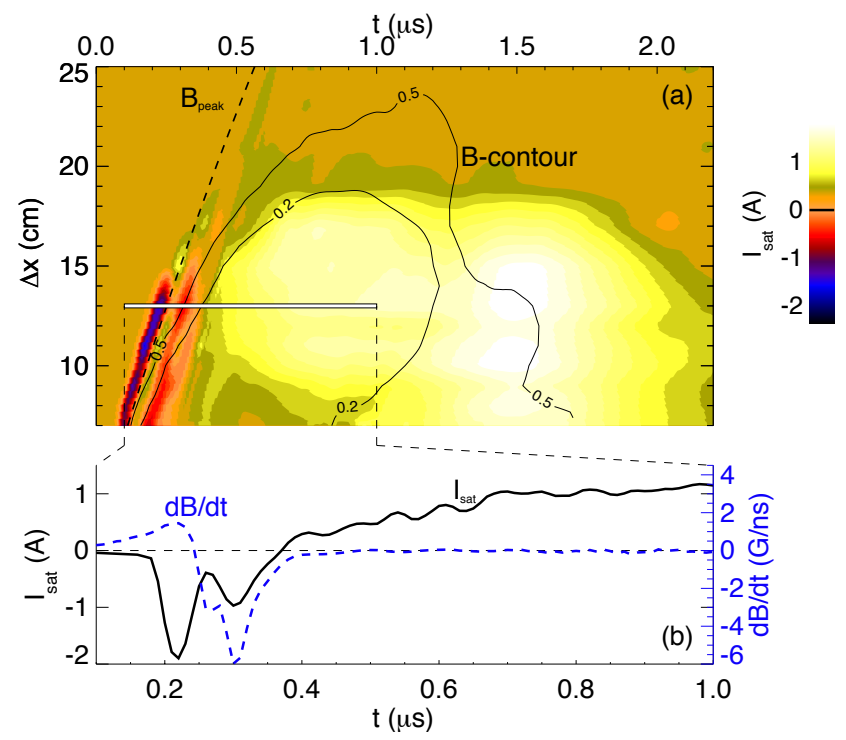

Fig 3: Ion-saturation-current measurements show electron heating at the edge of the diamagnetic cavity, and the blow-off plasma filling the bubble volume (a). The contours of the magnetic field as well as the time of peak compression from Fig. $2 b$ are shown for comparison. (b) Evolution of $I_{\text {sat }}$ and the Bdot-probe signal $13 \mathrm{~cm}$ from the target. The first negative spike in the Langmuir probe signal coincides with the time of peak- compression, while the second negative spike occurs during field expulsion. The Isat data also displays high frequency $(\sim 10 \mathrm{MHz})$ oscillations inside the bubble.

\section{I.4 Hybrid simulations}

The experiment was modeled with a two dimensional hybrid code from LANL. In the hybrid mode, the ions are treated as particles in self-consistently generated electromagnetic fields, while the electrons are modeled as an inertialess, charge-neutralizing fluid, thus eliminating the shortest length and time scales associated with the electron dynamics. The electron temperature is modeled to fluctuate adiabatically. Particles are tracked in two spatial coordinates ( $\mathrm{x}$ and $\mathrm{y}$, perpendicular to the external magnetic field) using three-dimensional velocities and fields. The simulation does not model the laser-plasma interaction explicitly but is initiated with a cloud of debris ions $\left(\mathrm{C}^{+4}\right)$ exploding at $\mathrm{M}_{\mathrm{A}} \sim 1.5$ isotropically into an ambient $\mathrm{He}^{+}$plasma at conditions 
consistent with the experiment $\left(\mathrm{n}_{0} \sim 9 \times 10^{11} \mathrm{~cm}^{-3}, \mathrm{~B}_{0} \sim 275 \mathrm{G}, \mathrm{T}_{\mathrm{i}} \sim 1 \mathrm{eV}, \mathrm{T}_{\mathrm{e}} \sim 5 \mathrm{eV}\right)$. A mean charge state of $\mathrm{C}^{+4}$ was determined by spectroscopic measurements performed in a related experiment at similar laser energies and is also consisted with radiation-hydrodynamic and collisional-radiative modeling.
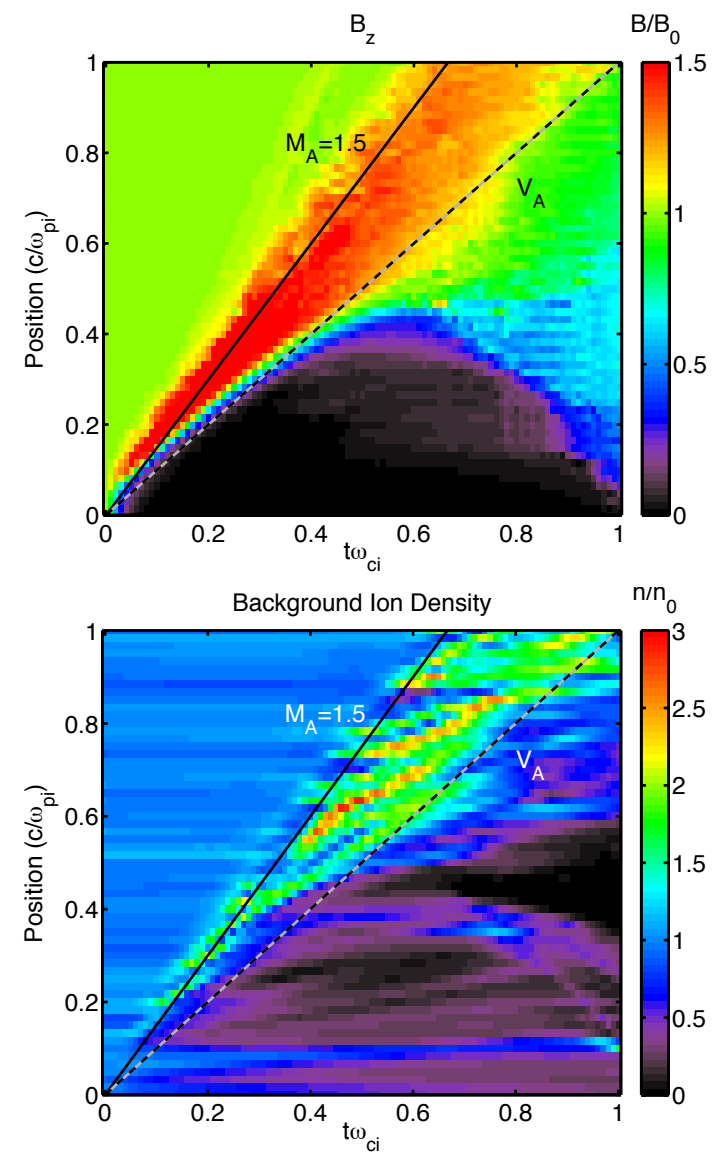

(a)

Fig. 4: Hybrid simulation of the laser-plasma expansion in the preformed plasma and magnetic field. (a) streak plot of the magnetic field profile during the first t $\omega_{c i}$ along the $x$ axis. A debris cloud containing $C^{+4}$ explodes spherically into a $\mathrm{He}^{+}$background in $275 \mathrm{G}$ from $x=0$ at $M_{A} \sim 1.5$. The formation of the diamagnetic cavity and magnetosonic pulse propagating away from the bubble are clearly visible. Plots (b) and (c) show the background and debris ion densities respectively.

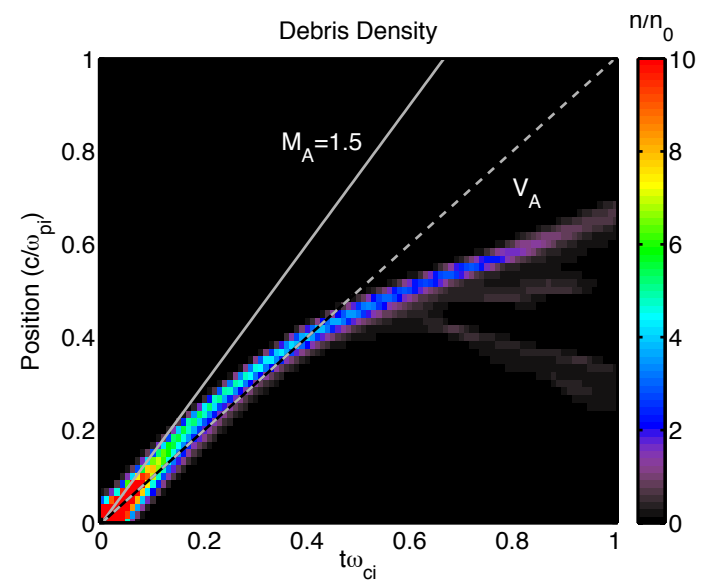

(b)

(c)

Figure 4 (a) shows the evolution of the magnetic field in a space-time streak plot, displaying $\mathrm{B}_{z}$ along $\mathrm{x}$ at a fixed $\mathrm{y}$ position $(\mathrm{y}=0)$ at various times. The exploding debris cloud is originally located in the center of the simulation box at $\mathrm{x}=\mathrm{y}=0$. The field-expulsion and compression, and the formation of the diamagnetic cavity is clearly visible. In the simulation the number of ions in the debris cloud was adjusted to create a bubble with a size of $\sim 0.45 \mathrm{c} / \omega_{\mathrm{pi}}(22 \mathrm{~cm})$, consistent 
with the experiment. Figure 4 (a) shows a magnetic field compression of $\sim 1.5$, which corresponds to the Alfvenic Mach number of the debris expansion and is consistent with the MHD- jump conditions. Figures 4 (b) and (c) shows streak plots similar to that of the magnetic field, but show the background and debris ion densities relative to $\mathrm{n}_{0}$, where $\mathrm{n}_{0}$ is the initial density of the $\mathrm{He}^{+}$background plasma. The background ions are pushed out of the diamagnetic bubble as they are accelerated by the piston. Figure 4 (c) shows that around $t \omega_{\mathrm{ci}} \sim 0.5$ some debris stagnates or turns around, and some moves outward at a sub-Alfvenic speed. It can be seen by these figures that the debris ions initiate a magnetosonic pulse which then separates from the debris cloud and propagates out radially; however, the pulse does not steepen into a shock under these conditions.

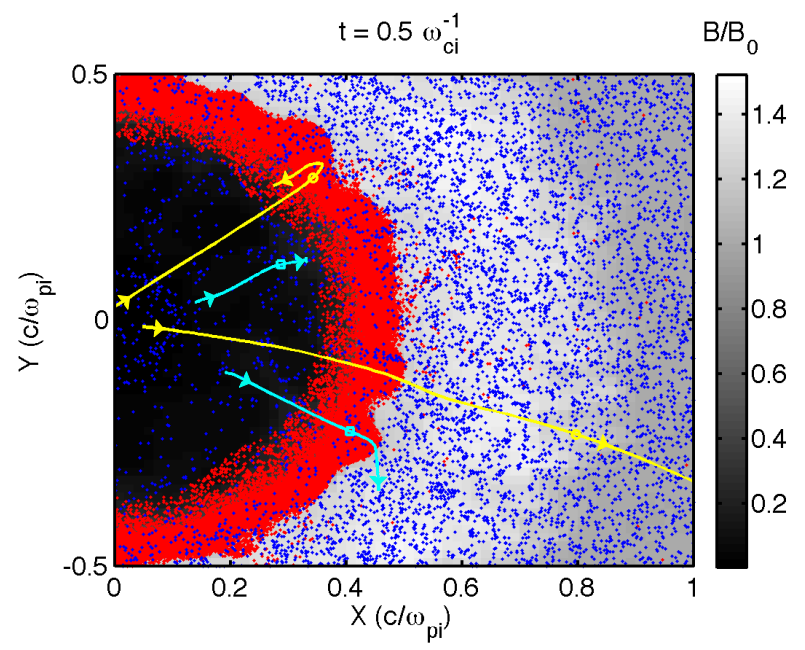

Fig 5: A composite image of the magnetic field strength $B_{z}$, a sampling of background ions (blue dots), a sampling of debris ions (red dots), along with trajectories of a couple of background (light blue) and debris (yellow) ion trajectories throughout the course of the simulation. The markers on the trajectories correspond to the test particle's current position at $t \omega_{c i}=0.5$.

When the piston stagnates at $t \omega_{\mathrm{ci}} \sim 0.5$, the bulk of the debris ions piles up at the bubble edge as shown in figure 5. The weak magnetosonic pulse can be seen just outside of the dense ring of debris ions. Complete magnetic field expulsion can be seen inside of the ring of debris ions. A few test particles were chosen to illustrate the motion of various background and debris ions. The yellow lines show two different debris ion particles, a fast ion which makes it out past the majority of the debris ions, and one that slows and diffuses back into the bubble at a later time. The light blue lines show two background $\mathrm{He}^{+}$ions, which clearly couple to the debris ions and move outward radially.

Fast shocks in space at these low Mach numbers $\left(\mathrm{M}_{\mathrm{A}} \sim 1.5\right)$ are observed, but the dissipation is usually only in the electrons. In the hybrid-model we use, there is effectively no resistivity or resistive heating. Ion reflection is the only dissipation mechanism, but the phase space (not shown) does not show clear evidence for reflected ions. It should be noted that adiabatic compression will only heat the background electron temperature to $\sim 7 \mathrm{eV}$. This suggests some other form of heating (e.g. anomalous, resistive) is responsible for the electron heating seen in the previous subsection. This is consistent with earlier experiments on magnetic pinches, which showed that only $20 \%$ of electron heating is due to adiabatic and Ohmic heating, while the rest is due to plasma turbulence.

It should be noted that the length scales within this simulation are less than the ion inertial length $\left(\mathrm{c} / \omega_{\mathrm{pi}}\right)$. The diamagnetic bubble radius is currently only $\sim 0.5 \cdot \mathrm{c} / \omega_{\mathrm{pi}}$. Since the hybrid implementation assumes the electrons are massless and high frequency electron effects are left out, length scales much less than the ion inertial length may be somewhat meaningless. Future experiments will take advantage of a new plasma source that supports higher densities in excess of $10^{13} \mathrm{~cm}^{-3}$ and a larger laser system that supports larger bubbles. Hybrid simulations using the 
larger ion density (smaller $\mathrm{c} / \omega_{\mathrm{pi}}$ ) show that a full-blown collisionless shock does indeed form at these improved ambient plasma conditions, with a diamagnetic bubble that spans multiple ion inertial lengths.

\section{I.5. EMISSION OF SHEAR-ALFVEN WAVES}

Figure 6a shows a typical magnetic flux probe signal of the transverse field component $\left(\mathrm{B}_{\mathrm{y}}\right)$ recorded $6.8 \mathrm{~m}$ axially from the target, at $(\mathrm{x}, \mathrm{y})=(12 \mathrm{~cm}, 0)$. A shear-Alfven pulse with an amplitude around $15 \mathrm{G}$ (or $\delta \mathrm{B} / \mathrm{B}=5 \%$ ) is clearly visible passing the probe around $20 \mu$ s after the laser is fired. We measure an axial field component $\delta \mathrm{B}_{\mathrm{z}}$ (dashed red line) that is around $1 / 10$ of $\mathrm{B}_{\mathrm{y}}$ (solid black line), characteristic of a shear wave. The horizontal field component $\left(\mathrm{B}_{\mathrm{x}}\right)$ at $\mathrm{y}=0$ is nearly zero (not shown). The frequency of the shear-wave is initially around $40 \mathrm{kHz}$ and below the cyclotron frequency $\left(0.4 \cdot f_{\mathrm{ci}}\right)$, corresponding to a wavelength of $8.3 \mathrm{~m}$. We measure field fluctuations as high as $70 \mathrm{G}$ (or $\delta \mathrm{B} / \mathrm{B}>25 \%)$ at the probe closest to the target $(\Delta \mathrm{z}=0.3 \mathrm{~m})$, decreasing to $40 \mathrm{G}$ (or $\delta \mathrm{B} / \mathrm{B}=15 \%$ ) at a distance of $1-1.5 \mathrm{~m}$, and to $20 \mathrm{G}$ at distances above $4 \mathrm{~m}$ (figure 7). These experiments where performed in the kinetic regime, as the Alfven speed is about half the thermal electron speed. Therefore, Landau damping could damp the wave and explain the decrease of the field magnitude with distance from the target. At later times (not shown), after several transit times of the wave, the signal is dominated by lower frequency oscillations due to standing waves in the $17 \mathrm{~m}$ long He-plasma column.

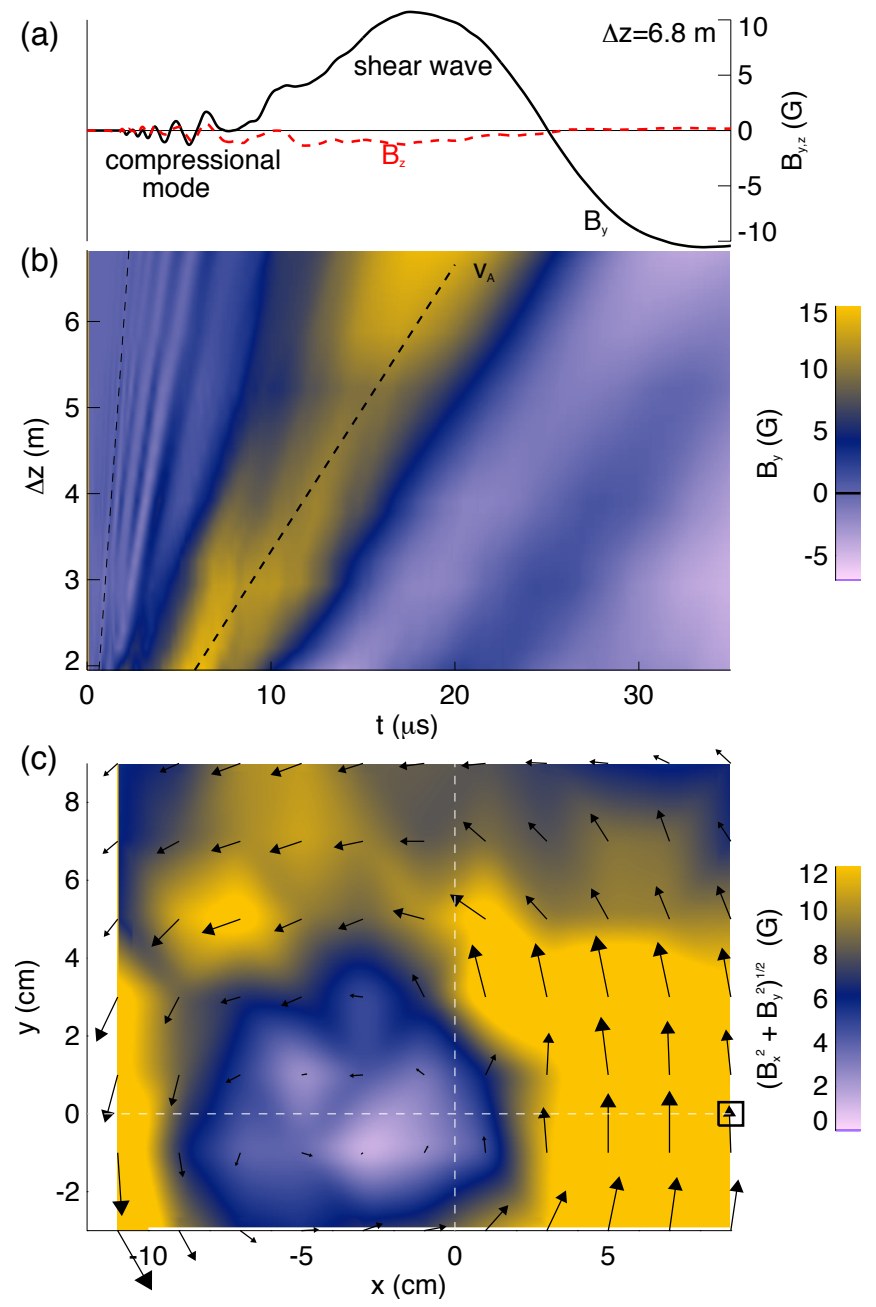

Fig 6: A shear-Alfven wave is launched by the diamagnetic bubble and propagates over several meters along the machine (z-axis) at $v A=3.3 \times 10^{7} \mathrm{~cm} / \mathrm{s}$. A fast wave procedes the shear-wave at $3 \times 10^{8} \mathrm{~m} / \mathrm{s}$, corresponding to electrons with a kinetic energy of $25 \mathrm{eV}$. (a) Evolution of $B_{y}$ and $B_{z}$ at $y=0, \Delta z=6.8 \mathrm{~m}$ from the target, and $x=9 \mathrm{~cm}$ from the center of the plasma column. (b) Contour plot of the trans-verse field component (By) as a function of time and spatial coordinate along the field (z). The plot is comprised of simultaneous measurements from 9 magnetic pickup coils distributed at longitudinal positions $\Delta z$ between $2 \mathrm{~m}$ and $6.8 \mathrm{~m}$. (c) Total transverse field component as a function of both transverse dimensions in a vertical plane at $\Delta z=6.8 \mathrm{~m}$ from the target at $t=20 \mu \mathrm{s}$. The coaxial current system is clearly visible and is aligned with the center of the plasma column. The arrows indicate the transverse direction of the magnetic field, while the rectangle at $(x, y)=(9,0)$ $\mathrm{cm}$ indi- cates the probe location for plots (a) and (b). 
Simultaneous measurements of this type with an array of 10 magnetic field probes distributed along the plasma column at distances $\Delta \mathrm{z}$ between 2 and $6.8 \mathrm{~m}$ from the target are shown in Fig. 6b. All probes were located in the horizontal (xz) plane through the plasma column center $(\mathrm{y}=0)$, at a transverse distance of $x=12 \mathrm{~cm}$ from the center of the machine where the wave-amplitude was close to the maximum. The traveling shear-Alfven wave is clearly visible, propagating along the magnetic field at $3.3 \times 107 \mathrm{~cm} / \mathrm{s}$, which is consistent with the Alfven speed computed at the plasma parameters. Figure $6 \mathrm{c}$ shows measurements of the transverse magnetic field components in the xy-plane at $\Delta \mathrm{z}=6.8 \mathrm{~m}$ from the target at the time when the shear-wave amplitude peaks ( 20 $\mu \mathrm{s})$. To obtain this data, one magnetic field probe was moved in two-dimensions over $20 \mathrm{~cm}$ horizontally and $12 \mathrm{~cm}$

The temporal evolution of $\mathrm{B}$ of transverse position (x-axis) at $\mathrm{y}=0$ is shown in Fig. 7. The quasicoaxial character of the current system centered at $\mathrm{x} \approx-3 \mathrm{~cm}$ is evident, with the maximum field amplitude $10 \mathrm{~cm}$ from the current channel. The current density that produces such a current system is shown in Fig. $8 \mathrm{~b}$ and is around $3 \mathrm{~A} / \mathrm{cm}^{2}$ and close to the electron saturation current. The total current carried by the wave is on the order of $1 \mathrm{kA}$. The rapid fall- off of the field outside the current-channel (faster than $\sim 1 / \Delta \mathrm{x}$ ) can only be explained with a negative return current around $1 \mathrm{~A} / \mathrm{cm}^{2}$ in a surrounding shell. In the shear-Alfven wave, the current along the magnetic field is carried by electrons, while the current across the field is carried by ions due to the ionpolarization drift. Although the data suggests that $I_{\text {enc }}$ vanishes at large $\mathrm{x}$, the spatial range covered in this measurement was not large enough to show that the net-current through any plane is exactly zero. In helium we measure shear-Alfven wave velocities of $650 \mathrm{~km} / \mathrm{s}$ and $1400 \mathrm{~km} / \mathrm{s}$ for magnetic fields of $600 \mathrm{G}$ and $1800 \mathrm{G}$, respectively. These velocities are consistent with the computed Alfven speed, considering that the plasma density increases from $8.5 \times 10^{11} \mathrm{~cm}^{-3}$ at 275 $\mathrm{G}$ to $2 \times 10^{12} \mathrm{~cm}^{-3}$ at $1800 \mathrm{G}$.

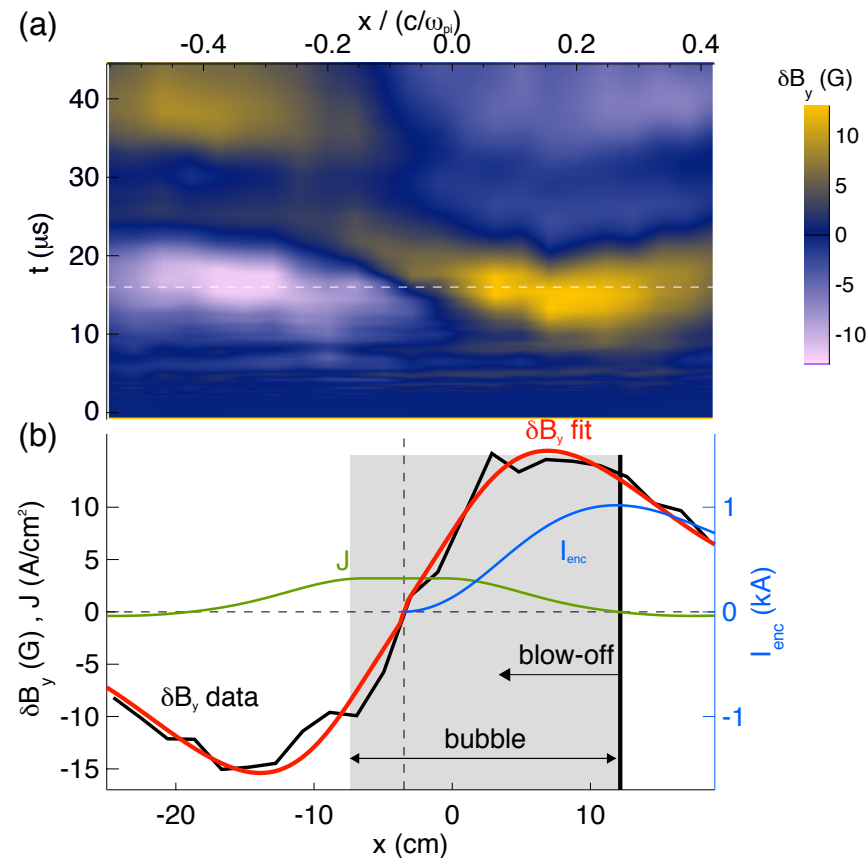

Fig 7: Evolution of the transverse magnetic field component $B_{y}$ at $y=0$ as a function of transverse position across the plasma column (x), at a distance of $\Delta z=5.2 \mathrm{~m}$ from the target in the axial direction (a). A shear-Alfven wave passes that probe roughly $15 \mu$ s after the laser-trigger, consistent with an Alfven speed of $330 \mathrm{~km} / \mathrm{s}$. A fastwave is also visible preceding the shear-wave. (b) Line-out of $B_{y}$ as a function of $x$ at the time when the peak of the pulse passes the probe $(t=15 \mu \mathrm{s})$. The shear-wave carries a total current around $1 \mathrm{kA}$ distributed over a $20 \mathrm{~cm}$ diameter channel, creating a traverse field up to $15 G($ or $\delta B / B=5 \%)$. We observe current densities around $3 \mathrm{~A} / \mathrm{cm}^{2}$ in the center with a return current around -1 $\mathrm{A} / \mathrm{cm}^{2}$ carried in a concentric shell. 
A fast wave is also observed, propagating along the plasma column at a wavefront velocity around $3 \times 10^{8} \mathrm{~cm} / \mathrm{s}$, corresponding to a beam of electrons with a kinetic energy of $25 \mathrm{eV}$. At an axial distance of $6.8 \mathrm{~m}$ from the target we observe low amplitude fluctuations of the magnetic field signal as early as $350 \mathrm{~ns}$ after the laser- trigger, corresponding to velocities in excess of $2 \times 10^{9} \mathrm{~cm} / \mathrm{s}$. These perturbations are due to the presence of a high-energy tail of supra-thermal electrons with $\mathrm{keV}$ energies. We observe field fluctuations of a few Gauss in all three orthogonal components. Figure 9 shows the auto spectrum of the flux-probe signal from Fig. 6a. The compressional mode is clearly visible at a frequency de- creasing from $1 \mathrm{MHz}$ at early times to $0.5 \mathrm{MHz}$ just before the arrival of the shear-wave.

\section{I.6. Coupling efficiency}

The coupling-efficiency to shear-Alfven waves can be estimated by integrating the measured magnetic-field profile $\mathrm{B}(\mathrm{r})$ over the volume of the current system. We find an energy $\mathrm{E}_{\mathrm{A}}$ around $0.9 \mathrm{~J}$, corresponding to a coupling efficiency of $5 \%$ from the laser to Alfven waves. The coupling efficiency from the diamagnetic cavity to the shear-wave is much higher at around $70 \%$. In addition, a magnetic energy around $50 \mathrm{~mJ}$ is contained in the compressional mode, which is too small to affect the total energy budget. Compared to earlier work at lower laser energies, we observe coupling efficiencies from the laser to Alfven waves an order of magnitude higher and shear-waves that contain an energy two-orders of magnitude higher. Field amplitudes in the present experiments are only an order of magnitude higher, since the width of the current system is also larger. Using higher background fields decreases the bubble size, and the current in the shear-wave decreases from $1 \mathrm{kA}$ at $275 \mathrm{G}$ to $600 \mathrm{~A}$ at $600 \mathrm{G}$ and $400 \mathrm{~A}$ at $1800 \mathrm{G}$. We thus observe the largest wave amplitudes and the highest cou- pling efficiency in helium at low fields. Experiments in neon yield smaller currents $(600 \mathrm{~A})$, and the total energy in the wave decreases to $300 \mathrm{~mJ}$. In experiments with varying magnetic field and ambient mass (table I), the frequency of the shear-wave is always around $0.5 \cdot f_{\mathrm{ci}}$ over a large range of gyro-frequencies between $21 \mathrm{kHz}$ for neon in $275 \mathrm{G}$ to $685 \mathrm{kHz}$ for helium in $1800 \mathrm{G}$. The width of the current system (as measured from maximum to minimum field) decreases with the ambient field from $20 \mathrm{~cm}$ at $275 \mathrm{G}$ to 10 $\mathrm{cm}$ at $1800 \mathrm{G}$, as the diameter of the diamagnetic cavity shrinks from $20 \mathrm{~cm}$ to $5 \mathrm{~cm}$. We measure no difference in the coupling to shear-waves between shots with fast $\left(\mathrm{M}_{\mathrm{A}}=1.5\right)$ and slow $\left(\mathrm{M}_{\mathrm{A}}=1.1\right)$ laser-blow-offs, even though there is more electron heating at the edge of the bubble at higher speed. The observed reduction in coupling efficiency at higher fields is consistent with earlier work at less energ that found better coupling efficiencies where the cavity lifetime $\tau \mathrm{B}=$ $1 /\left(4 \mathrm{f}_{\mathrm{B}}\right)$ is closer to the cyclotron frequency $\left(\mathrm{f}_{\mathrm{B}} / \mathrm{f}_{\mathrm{ci}} \rightarrow 1\right)$. This is equivalent to evaluating the bubble size $D_{B}$ relative to the Alfven wavelength $D_{B} / \lambda_{A}$, where $\lambda_{A} \sim v_{A} / f_{c i} \sim \sqrt{m i}$. Thus $D_{B} / \lambda_{A} \sim$ $\left(E / B^{2}\right)^{1 / 3} /{ } m_{i}$ increases with $E$ but decreases with $B$ and the ambient ion mass mi. In helium at $275 \mathrm{G}$, the bubble expansion time is $\tau \mathrm{B} \approx 1 \mu \mathrm{s}$ and the ratio is $\mathrm{f}_{\mathrm{B}} / \mathrm{f}_{\mathrm{ci}} \approx 2$. At1800 $\mathrm{G}$, the ratio increases to 4. Similarly, the coupling to Alfven waves decreases as the ambient mass increases.

\section{Collisionless shock experiments at the LANL Trident Laser}

Since ambient densities in the LAPD are currently still limited to $10^{12} \mathrm{~cm}^{-3}$ by the barium-oxide coated cathode, we performed a laser-only experiment at the LANL Trident facility at higher densities. The experiment demonstrates key elements in the production of laser-driven, magnetized, laboratory-scaled astrophysical collisionless shocks. These include the creation of a novel magnetic piston to couple laser energy to a background plasma and the generation of a collisionless shock precursor. We also observe evidence of decoupling between a laser-driven fast ion population and a background plasma, in contrast to the coupling of laser-ablated slow ions with background ions through the magnetic piston. 2D hybrid simulations further support these 
developments and show the coupling of the slow to ambient ions, the formation of a magnetic and density compression pulses consistent.

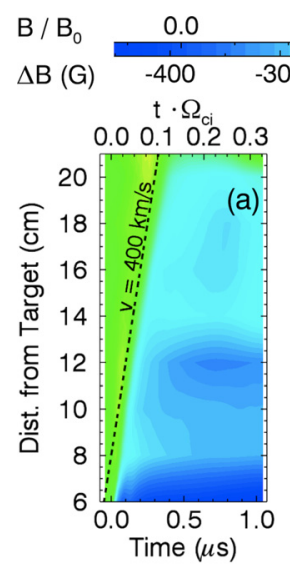

$-2$

0.5

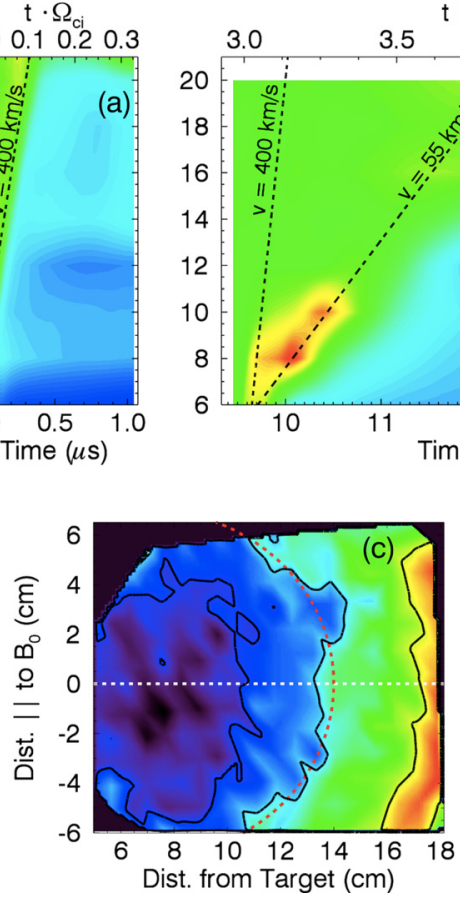

1.0

$0: \frac{1}{i}: \frac{1}{100} 200$

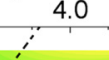

4.5

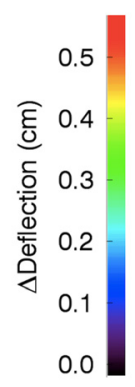

Fig. 8: (a) and (b) Magnetic contour plots of the heater-and driver-created diamagnetic cavities for a background field $B_{0}=390 \mathrm{G} . w_{c i}$ is the ion cyclotron frequency. The heater pulse arrives at $t=0 \mathrm{us}$, and the driver pulse arrives at $t$ $=9.5$ us. (c) Proton deflection contour plot of the heater-driven diamagnetic cavity 0.6 us after the heater pulse for $B_{0}$ $=800 \mathrm{G}$.

Like previous efforts, we use lasers to define the shock geometry (strictly perpendicular, though the transition to other geometries would be possible) and to create an exploding plasma. However, rather than directly shocking a background plasma, the exploding plasma creates a magnetic piston that accelerates background plasma, which in turn shocks unperturbed background plasma. Furthermore, unlike typical laser experiments, these experiments are largescale $(\sim 50 \mathrm{~cm})$, letting the piston interact with the background plasma while still allowing sufficient space and time for a shock to form. We characterize the process using magnetic flux probes, optical Thomson scattering, emission spectroscopy, and proton deflectometry. The experiments were performed at the Trident laser facility at Los Alamos National Laboratory. A graphite target was placed in vacuum at the inner edge of a $50 \mathrm{~cm}$ diameter pulsed Helmholtz coil that could create a quasi-static external magnetic field up to $1 \mathrm{kG}$. The magnetic field was aligned parallel to the central axis of the coil and defines the $\mathrm{z}$ axis, while the normal to the target surface defines the $\mathrm{x}$ axis. The target could be rotated or translated for each shot to provide a fresh surface and was irradiated by two sequential laser pulses. The first "heater" beam $(1053 \mathrm{~nm}, 50 \mathrm{~J}$, $50 \mathrm{~ns}, 1: 5,10^{11} \mathrm{~W} / \mathrm{cm}^{2}$ ) created an ambient, low-density, magnetized carbon plasma. The second "driver" beam (1053 nm, $\left.250 \mathrm{~J}, 5 \mathrm{~ns}, 6: 5,10^{12} \mathrm{~W} / \mathrm{cm}^{2}\right)$ ablated a super-Alfvenic blow-off plasma, which subsequently created a magnetic piston that accelerated the ambient plasma into a shock front. A modular array - consisting of 40 probe slots spaced $1 \mathrm{~cm}$ apart—of single-axis, non-differential $1 \mathrm{~mm}$ magnetic flux ("bdot") probes was aligned along x. The probes measured up to $40 \mathrm{~cm}$ from the target the $\mathrm{z}$ component of the magnetic field compression, expulsion, and diffusion of the expanding laser-plasma (all references to magnetic field hereafter refer solely to 
the $\mathrm{z}$ component, unless otherwise stated). A third beam line could be configured as a Thomsonscattering probe $(527 \mathrm{~nm}, 0.5 \mathrm{~ns}, 50 \mathrm{~J})$ to measure electron density and temperature, or as a highintensity, short-pulse $\left(200 \mathrm{TW}, 10^{19} \mathrm{~W} / \mathrm{cm}^{2}, 500 \mathrm{fs}\right)$ probe for proton deflectometry to map magnetic fields. Either probe could be arbitrarily delayed relative to the heater or driver beam.

The ablation of the target by the heater beam creates a diamagnetic cavity as the blow-off plasma expands outward. This Hall physics extension of MHD on small spatial scales, where the gyroradii of electrons and ions differ, is well known and has been studied explicitly in diamagnetic cavity formation related to barium releases in space. Furthermore, because the magnetic compression is closely tied to the ablated ions, its speed can act as a proxy for the speed of the (leading) ions (in fact, simulations show that the driving ions move somewhat faster than the corresponding compression). The magnetic compression ahead of the cavity moves at a speed of $400 \mathrm{~km} / \mathrm{s}$ (the initial speed of the blow-off plasma), while the cavity reaches a maximum size of $\sim 20 \mathrm{~cm}$ (estimated where the magnetic field returns to its initial value). In general, the cavities in vacuum at various laser energies were quasi-spherical and homogeneous, and their sizes were consistent with the scaling provided by the conservation laws for the magnetic stopping radius (assuming the relative energy partition between shots was consistent). As a result, the extent of the ambient plasma was assumed to be the cavity size. After 7, the magnetic field was measured to have completely diffused back in (i.e., returned to its initial value, though much faster than the classical Bohm diffusion time of 300 us).

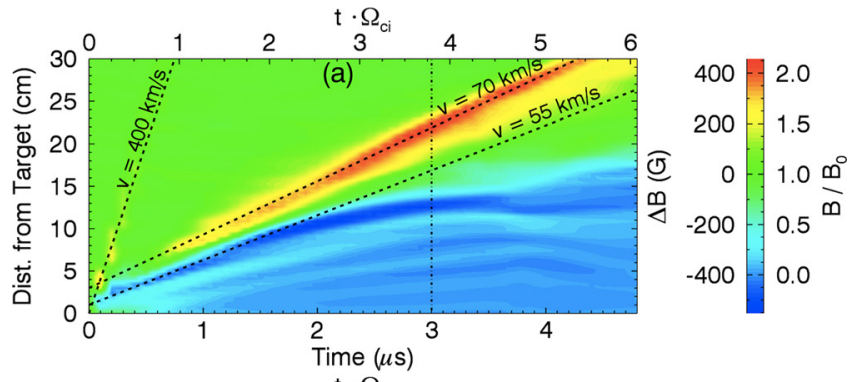

Fig. 9: (a) Magnetic contour plot and (b) ambient ion density (with overlaid piston ion density) contour plot from a $2 D$ hybrid simulation of the drivercreated diamagnetic cavity for a

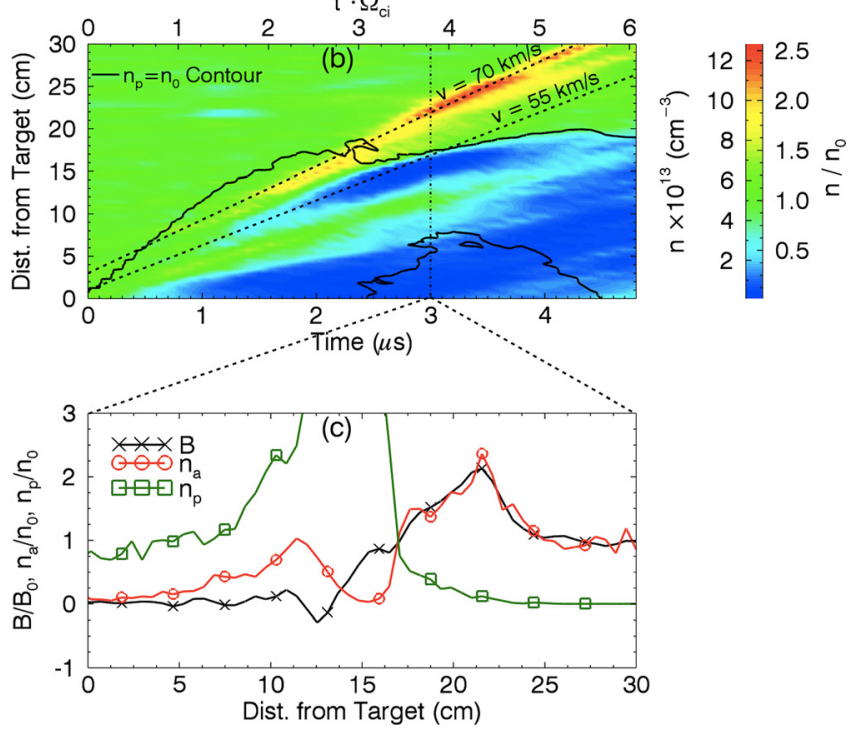
background field $B_{0}=390 \mathrm{G}$ and initial ambient ion density $n 0=5 \times 10^{13} \mathrm{~cm}^{-3}$. The driver pulse arrives at $t=0$. As in Fig. 1, a fast ion population moving at $400 \mathrm{~km} / \mathrm{s}$ decouples from the background, while a slow ion population at $55 \mathrm{~km} / \mathrm{s}$ forms a cavity before stagnating. The compression ahead of the cavity moving at $70 \mathrm{~km} / \mathrm{s}$ indicates that the ambient ions have been swept out of the cavity and accelerated. (c) Normalized profiles at $t=3$ us for magnetic field $B$ (crosses), ambient density (circles), and piston density (squares).

A simulation of the driver component of the experiment was carried out using a 2D hybrid code with massless fluid electrons and kinetic ions The simulation did not include the laser-target interaction, but focused on a dual-population plasma (fast and slow components) expanding out 
from a planar target perpendicular to a uniform magnetic field into an ambient plasma at the experimental conditions. The salient features observed in the experiment, including the formation of a diamagnetic cavity by the piston ions, the coupling of the piston to the ambient ions, the formation of a magnetic and density compression pulse consistent with a collisionless shock, and the decoupling of the fast ions, were also observed in the simulation (see Figs. 9(a) and 9(b)). In particular, Fig. 9(b) shows that the piston ions stagnate inside the diamagnetic cavity while the ambient ions are swept out, consistent with Larmor coupling between the piston and background plasma. Additionally, Fig. 9(c) shows both the ambient ion density and the magnetic field being compressed by a factor of 1.5 , consistent with the experimentally derived Mach number and the jump conditions for a collisionless shock.

These experiments have demonstrated that a laser-ablated plasma can create an effective magnetic piston that couples laser energy to a background plasma, as evidenced by the reduction in diamagnetic cavity size. This coupling has generated features that are consistent with a low Mach number magnetized collisionless shock. 2D hybrid simulations further show that under the experimental laser and plasma conditions, a laser-facilitated magnetic piston sweeps up background plasma that steepens into a collisionless shock as it expands into the non-perturbed ambient plasma. In addition, we see evidence of decoupling between fast ions and the background plasma. While future experiments will refine our ability to create and diagnose collisionless shocks, more importantly the use of a laser-generated magnetic piston will allow us to transition to the quasi-parallel shock geometries prevalent in space plasmas but never achieved in a laboratory setting.

\section{High-energy laser facility}

A new kilojoule-class laser has been activated at UCLA for experiments on laser-driven shocks. The laser can deliver energies up to $500 \mathrm{~J}$ in a $25 \mathrm{~ns}$ pulse at $1053 \mathrm{~nm}$, and potentially up to $1.5 \mathrm{~kJ}$ at longer pulses ( 100-1000 ns). The project was funded by the American Recovery and Reinvestment Act through a supplement to this DOE/NSF partnership in basic plasma science grant. The laser includes recycled components from the decommissioned NOVA laser of LLNL and was designed as a university-scale system using modern multi-pass architectures along with a trimmed-down pulsed power configuration to reduce the footprint and project cost. Our laser system is now the world's highest-energy university laser, the only kilojoule class laser solely dedicated to basic science, and the only laser of this magnitude ever designed, built, and operated by graduate students!

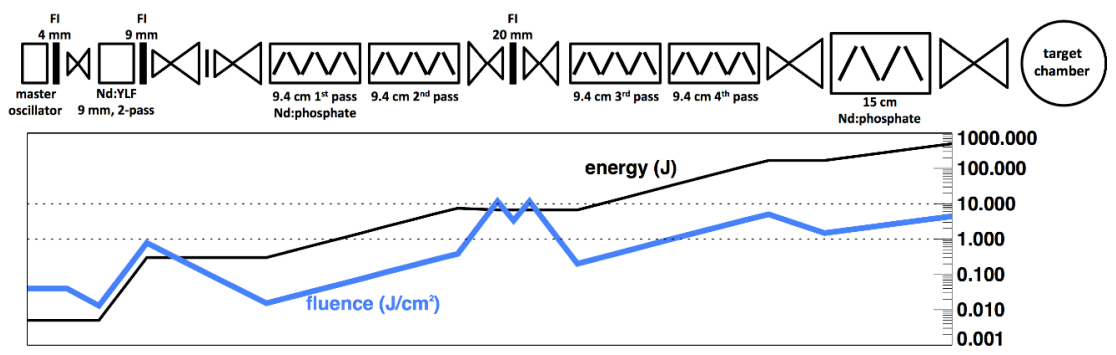

Fig 10: Block-diagram of the different amplifier stages, key optical components, and the variation of pulse energy and fluence throughout the laser chain.

The optical-layout is configured in a classical master-oscillator power amplifier (MOPA) architecture (figure 10), including a Nd:YLF front-end, and a series of flashlamp-pumped rod and 
Brewster disk amplifiers driven by a $500 \mathrm{~kJ}$ capacitor bank (equivalent to the pulsed power of the Texas petawatt laser). While the total length of the optical system is $45 \mathrm{~m}$, we use a folded architecture that fits on three 10' x 3' optical tables.

The master oscillator is currently comprised of a $4 \mathrm{~mm} \mathrm{Nd:YLF} \mathrm{rod} \mathrm{amplifier} \mathrm{in} \mathrm{a} \mathrm{Q-switched}$ cavity that includes a frequency selective $30 \mathrm{~mm}$ solid fused silica etalon. Inside the resonator, a low power oscillation is allowed to build over many passes through the etalon (over 1-2 $\mu \mathrm{s}$ ) resulting in a single longitudinal mode. This weak single frequency oscillation then serves as a seed to build up a high power Q-switched pulse. The pulse width can be varied between $8 \mathrm{~ns}$ and several tens of ns by changing the gain in the Nd:YLF rod at the time of switching. The $1053 \mathrm{~nm}$ transition in Nd:YLF closely matches the gain curve peak in the Nd-doped phosphate glass used in the large aperture disk amplifiers. A single frequency master pulse $\left(\delta \mathrm{f} / \mathrm{f}<10^{-6}\right)$ is employed to assure reproducible smooth temporal pulse shapes without modulation from multiple longitudinal

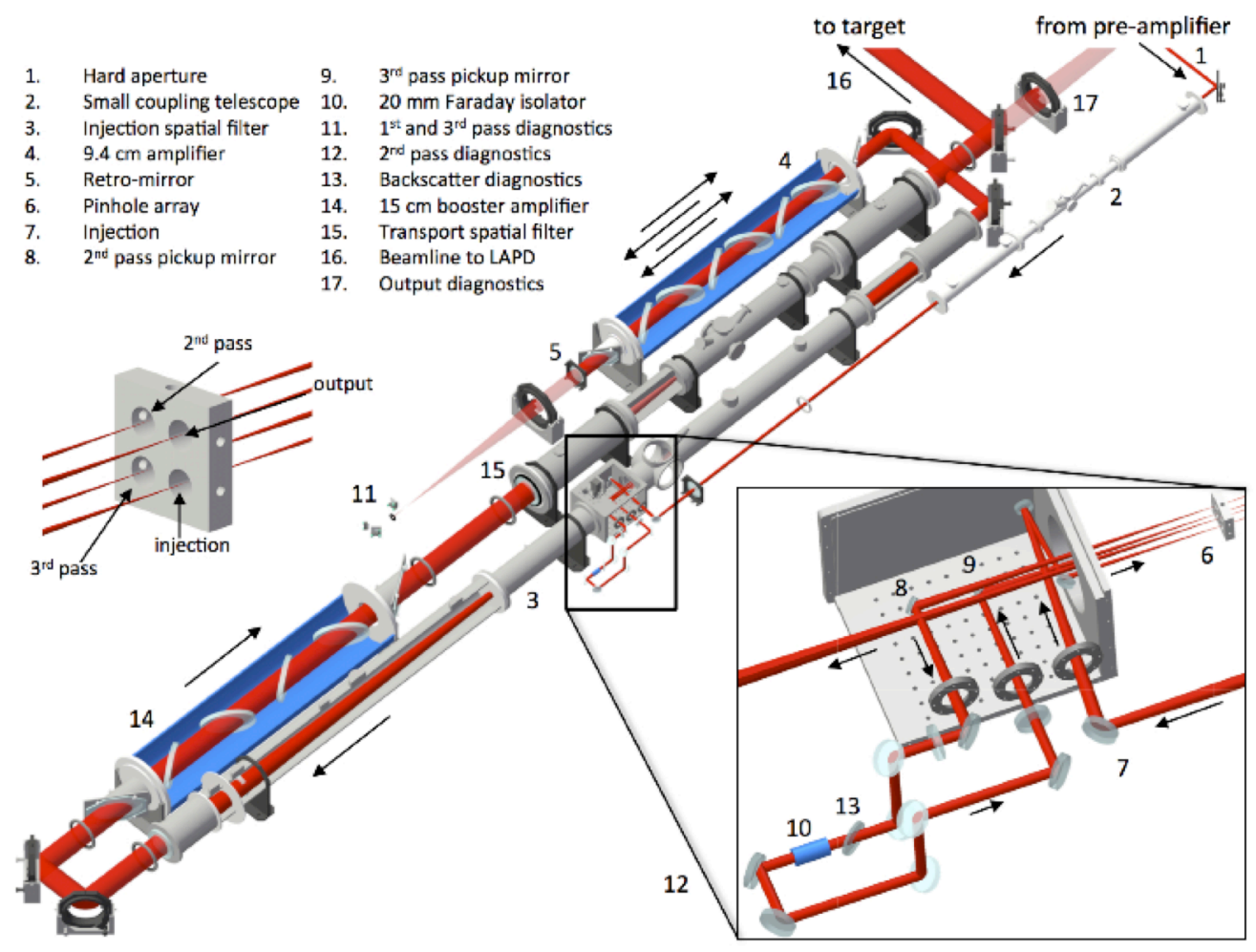

Fig 11: Optical layout of the power-amplifier section, including the injection spatial filter for angular multiplexing, the two Brewster disk amplifiers and the transport telescope to the target. The close-up in the lower-right corner shows the geometry used for far-field separation and the final Faraday rotator.

modes. The output from the mono-mode oscillator is around $5 \mathrm{~mJ}$ and is pre-amplified to a few $100 \mathrm{~mJ}$ in a flashlamp-pumped $9 \mathrm{~mm} \mathrm{Nd:YLF} \mathrm{rod} \mathrm{in} \mathrm{a} \mathrm{double-pass} \mathrm{configuration.}$

A large $(6 \mathrm{~m}, \mathrm{f} / 36)$ vacuum relay telescope is used for an angle-multiplexed four-pass through the $9.4 \mathrm{~cm}$ main amplifier (Fig. 11). We inject the low energy pulse from the pre-amplifier $(250 \mathrm{~mJ})$ into this large spatial filter using a high-reflector positioned $28 \mathrm{~cm}$ from the focus. The beam is collimated at a reduced diameter of $50 \mathrm{~mm}$ in the first two passes through the $9.4 \mathrm{~cm}$ head to simplify alignment, since the fluence is low. A retro-reflector behind the first pass located in the 
relay plane sends the beam back through the amplifier and into the large spatial filter at a small angle of $0.5^{\circ}$ relative to the first pass. The spatial filter pinholes for different passes are separated by $2 \mathrm{~cm}$ vertically and horizontally, which leads to a maximum beam separation of $3 \mathrm{~cm}$ on the small $(\mathrm{f}=2: 34 \mathrm{~m})$ telescope lens. A second pickup mirror located $40 \mathrm{~cm}$ from the pinhole ejects the beam from the spatial filter, where it is re-collimated to a demagnified beam of $15 \mathrm{~mm}$ diameter. The energy after the second pass is $6.8 \mathrm{~J}$, corresponding to a fluence of $10 \mathrm{~J} / \mathrm{cm}^{2}$ at the pickup mirror and $12 \mathrm{~J} / \mathrm{cm}^{2}$ on the exit port window. These fluences are the highest in the system but are a factor of two below the manufacturers specified damage threshold. We maintain a clearance of $7 \mathrm{~mm}$ between each beam and the edges of the pickup mirrors. A permanent magnet Faraday isolator with high damage threshold thin film polarizers and $20 \mathrm{~dB}$ isolation is installed in the relay plane and is the last isolator before the target. The beam is then injected back into the relay telescope by a third pickup mirror located $34 \mathrm{~cm}$ from the far-field for the third and fourth pass. The nominal output energy after the last $9.4 \mathrm{~cm}$ pass is $167 \mathrm{~J}$, which is well below the $850 \mathrm{~J}$ saturation limit of the amplifier. The fluence of $5 \mathrm{~J} / \mathrm{cm}^{2}$ is a factor of 2 below the damage threshold of the laser disks due to platinum inclusions at this pulse length. The large injection spatial filter image-relays the beam onto the $15 \mathrm{~cm}$ booster amplifier, where it is amplified to 500 $\mathrm{J}$. A total energy of $1.5 \mathrm{~kJ}$ is stored in the four $15 \mathrm{~cm}$ disks, well above the final output energy. Photograph of the main-amplifier and the pulsed-power bank are shown in figures 22 and 23.
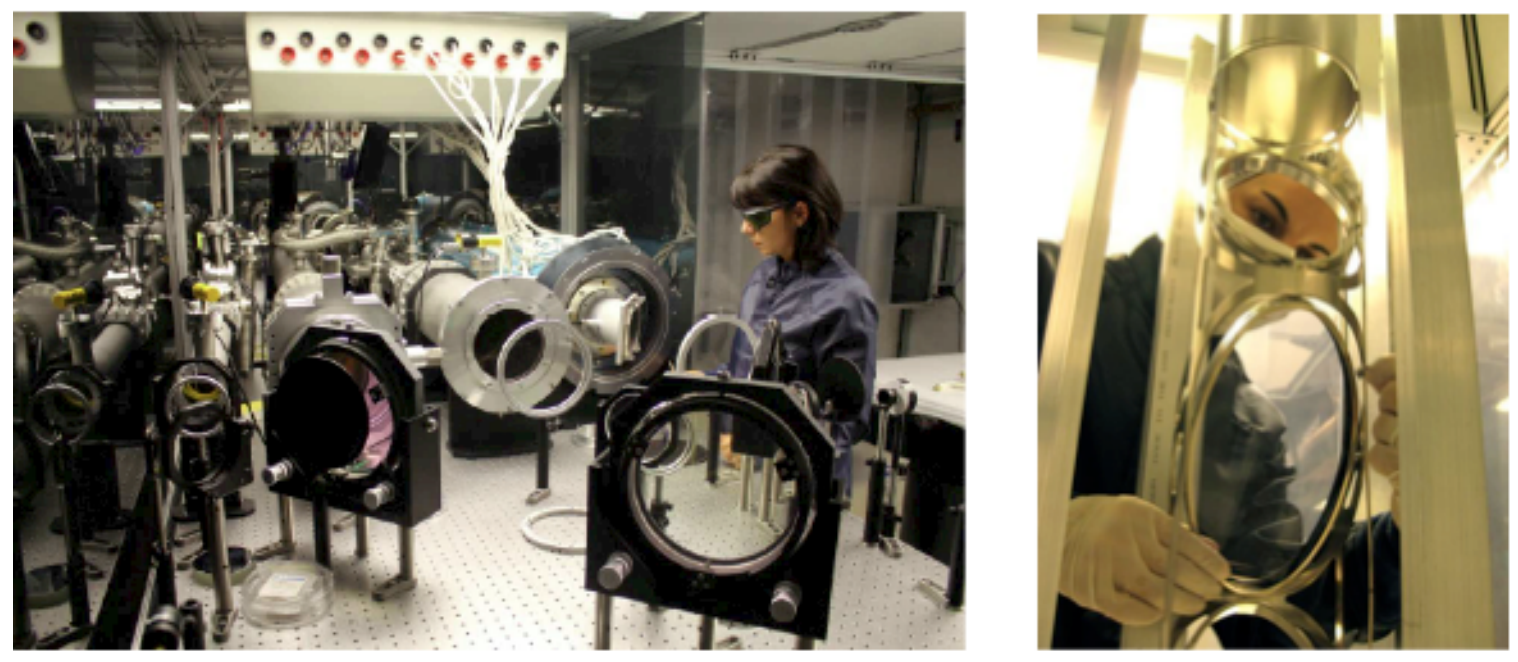

Fig 12: Left: Photograph of the $9.4 \mathrm{~cm}$ four-pass main-amplifier and the transport spatial filter. The $15 \mathrm{~cm}$ booster amplifier on the far side of the laser-tables is not visible. Right: the $10 \mathrm{~cm}$ NOVA Brewster amplifier disks being assembled in the amplifier structure (bird-cage). UCLA is the only institution that has successfully assembled a cylindrical NOVA amplifier in-house and without the dedicated NOVA assembly fixture available at LLNL. A $18 \mathrm{ft}$ tall class 10,000 clean-room was built at UCLA with a vertical amplifier assembly station to allow refurbishing of the NOVA amplifiers.

The NOVA phosphate disks are hygroscopic and had to be resurfaced since they were not kept in a controlled environment for several years. In order to protect the glass from humidity we purge each amplifier with dry air at 30 standard cubic feet per hour (SCFH) at all times to maintain a positive pressure in the hermetically sealed housings. Large aperture Brewster windows are installed at both ends of the amplifier housing for beam entry. Dry air is produced on site by a compressed air dryer, which delivers $390 \mathrm{SCFH}$ at $-100^{\circ} \mathrm{F}$ dew point at the 60 psig compressed air pressure. The air flow is temporarily stopped for a laser shot and is increased to $300 \mathrm{SCFH}$ for 
15 minutes after the shot to speed up cooling of the lamps and disks. Flow rates through the disk and lamp chambers are adjusted to be identical to avoid stress on the cylindrical pyrex blast shield that separates the lamp chamber from the disk chamber. We replaced the original silver plated reflectors from NOVA (80\% reflectivity) with highly reflective aluminum ( $>98 \%$ reflectivity) which does not tarnish due to the oxygen in the dry air purge. Instead of the shaped single-cusp reflectors from the original NOVA design, we use a simple cylindrical reflector geometry.

The two disk amplifiers are driven by a $500 \mathrm{~kJ}$ capacitor bank with high-current ignitron switches, configured similarly to the original NOVA design. The $9.4 \mathrm{~cm}$ amplifier contains 16 flash tubes connected to pulse forming networks (PFN) in 12 pairs of in-series connected lamps. The $15 \mathrm{~cm}$ amplifier contains 24 flash tubes. Each lamp pair is driven by two paralleled $52 \mu \mathrm{F} /$ $22 \mathrm{kV}$ capacitors in one circuit. The entire bank consists of 20 such circuits with a total capacitance of $2 \mathrm{mF}$ that stores $40 \mathrm{C}$ at $20 \mathrm{kV}$. Each PFN includes a $450 \mu \mathrm{H}$ inductor connected in series with the capacitors to stretch the current pulse length to more than $1 \mathrm{~ms}$ and limit the current to $4 \mathrm{kA}$. The resistivity of the flash lamps damps the circuit so that only a small reverse current appears at the capacitors and the high-current switch. The capacitor housings are isolated from the support frame by polyvinyl chloride sheets (PVC) and connected to pulsed power ground via $15 \Omega$ tube resistors. Each frame is electrically isolated from the ground and connected to pulsed power ground via $100 \Omega$ disc-resistors, which can dissipate $135 \mathrm{~kJ}$ in case of accidental arcing. Each circuit includes a high current fuse (4400 A $\left.{ }^{2} \mathrm{~s}\right), 3.9 \mathrm{k} \Omega$ charge resistor, and a shorting switch with $5 \Omega$ dump resistor for overvoltage protection. Each capacitor pair can be safely discharged in less than a second through a $1 \mathrm{k} \Omega$ disc resistor with a pneumatic dump-switch. We employ a separate high current switch for the $9.4 \mathrm{~cm}$ bank ( 8 circuits) and the $15 \mathrm{~cm}$ bank (12 circuits). Thus each amplifier can be fired independently. Following the original NOVA design we use a dual-ignitron switch for each bank with two ignitrons in series to prevent premature breakdown. A capacitive voltage divider consisting of two $10 \mathrm{M} \Omega$ tube resistors paralleled by 100 $\mathrm{nF}$ capacitors ensure equal division of the supply voltage across the two ignitrons. The ignitrons are water cooled to $17^{\circ} \mathrm{C}$ with a closed loop de-ionized water chiller. We monitor and maintain a conductivity below $20 \mu \mathrm{S} / \mathrm{cm}$, which corresponds to an additional resistance of $20 \mathrm{M} \Omega$ per ignitron in parallel with the voltage divider caused by the $1.2 \mathrm{~m}$ long water hose. Each anode is heated with a $60 \mathrm{~W}$ ceramic emitter at a distance of $15 \mathrm{~cm}$ to prevent condensation of the mercury. We maintain a minimum temperature difference of $10 \mathrm{oC}$ between anode and cathode at all times to guarantee a hold off voltage of $20 \mathrm{kV}$. Ignitrons are triggered by a commercial fourchannel trigger generator using only one of the ignitor pins per ignitron. The igniters are galvanically decoupled from the trigger board using high current transformers. Pulsed power bank and ignitron switches are installed in a $130 \mathrm{ft}^{2}$ pulsed power cave with the ignitron switches mounted on free-standing support structures (so called NOVA brass giant), which define pulsed power ground and are electrically insulated from the floor. Power transmission between the capacitor cave and the amplifiers is facilitated by $15 \mathrm{~m}$ long RG-217 coaxial cables routed on fiber-glass trays. An additional RG217 cable is installed between the laser head inner reflector of each amplifier and the pulsed power ground at the ignitrons to minimize current though the building ground in case of explosive flash lamp failure. Pulsed power ground and building ground are connected through a $100 \Omega$ resistor at only one point near the brass giant. Both banks for the two amplifiers are charged with the same $8 \mathrm{~kJ} / \mathrm{s}(25 \mathrm{kV})$ power supply in 45 seconds. A freewheeling diode assembly with $100 \Omega$ series resistors $(100 \mathrm{~W})$ protects the PSU from an accidental breakdown. In case of voltage reversal a high-voltage diode $(8 \mathrm{~A}$ at $48 \mathrm{kV}, 1.5 \mathrm{kA}$ surge current) will discharge the entire bank through a high power $100 \Omega$ disc-assembly resistor which can dissipate close to a MJ of energy. A small capacitor $(150 \mathrm{nF}, 25 \mathrm{kV})$ with $100 \mathrm{M} \Omega$ bleeding resistor connected to the PSU output protects the unit from an accidental open load condition. Three normally open high-voltage relays isolate the ground and the high-voltage terminals for both banks when charging is complete, immediately before the discharge trigger. 
Completely isolating the PSU from the pulsed power bank for the discharge protects the unit from voltage reversal and ensures controlled separation of the pulsed power and building ground. Once the PSU is disconnected, the capacitor banks slowly discharge through the voltage divider and cooling water in the ignitrons ( $\mathrm{RC}=3.6$ hours). We use a field programmable gate array (FPGA) to automatically control the charging sequence, including the opening of the relays to keep this time to a minimum $(10 \mathrm{~ms})$ and reproducible from shot to shot. In the charging sequence the PSU high-voltage output is disabled upon full charge shortly before the charge relays open. We use original NOVA energy storage capacitors, ignitrons, and charge resistors but replaced the inductors, dump switches and dump resistors, as well as the high current fuses. We use original NOVA flash tubes with a length of 44" and xenon fill at 300-700 torr with a light conversion efficiency up to $50 \%$. Although the pulsed power bank and lamps are qualified for a nominal bank voltage of $22 \mathrm{kV}$ the optical system was designed for reduced amplifier gains. We therefore operate the lamps at $16 \mathrm{kV}$ to increase their lifetime without the need of a low-energy preionization discharge.
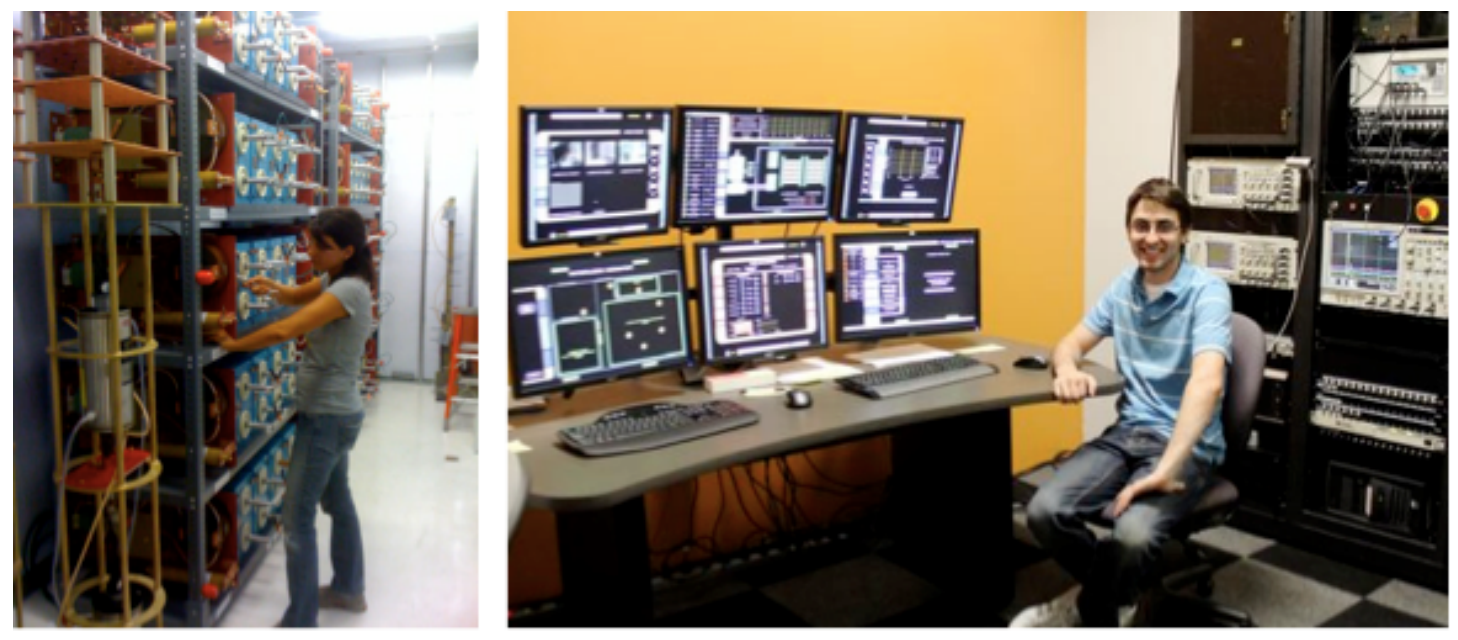

Fig 13: Left: UCLA researcher Carmen Constantin assembling the $500 \mathrm{~kJ}$ capacitor bank with high-current ignitron switches that power the flashlamps. The computer-control system (right picture) was built from scratch by a UCLA graduate student (Derek Schaeffer) and controls more than 500 input/output channels for pulsed-power, laser-beam-diagnostics, safety interlock systems, and the target area (https://decibel.ni.com/content/groups/sweet-apps/blog/2012/03/06/ ).

All laser and pulsed power systems are controlled by an array of stand-alone $400 \mathrm{MHz}$ FPGAs (National Instruments, CompactRIO ${ }^{\mathrm{TM}}$ ) connected to each other through a secure $100 \mathrm{Mbps}$ intranet based on plastic- optical-fiber (POF). Personal computers connected to this intranet only monitor the FPGA status through network shared variables but do not control any crucial or time sensitive systems. Areas in the laboratory that are physically separated (pulsed power cave, laser tables, target area, control room, LAPD) use individual FPGAs for data acquisition and control. The control system consists of more than 300 input/output channels including fast digitizers for laser-pulse shape, flash lamp current and beam energy measurements, CCD cameras for beam diagnostics, and stepper motor controllers for spatial filter pinhole and wave plate adjustments. A separate FPGA is used for a laser-safety interlock that connects to sensors and controls throughout the laboratory via plastic fibers. Pulsed power diagnostics include a self-powered high-voltage to frequency converter board on each ignitron switch, coupled to the pulsed-power- 
FPGA with plastic fiber senders and receivers to measure the actual voltage on each bank. The discharge current through each lamp pair and the reflector return cables are measured with current transformers coupled to $12 \mathrm{bit}, 100 \mathrm{kS} / \mathrm{s}$ digitizers on the FPGA. Beam parameters are monitored throughout the system by diagnosing low-power leaks through the retro- reflector and turning mirrors. Near-field and far-field are recorded on 12-bit charge-coupled-device (CCD) cameras. An aspheric f/10 lens relay-images the retro-mirror plane onto two cameras for near- field measurements of the first and third pass. The leaks from the two passes are separated at the farfield, where the beams are offset by $8 \mathrm{~mm}$. Similarly, the second pass is monitored behind the 20 $\mathrm{mm}$ rotator, and the final output behind the transport telescope. At each location both near-field and far-field are recorded on the same CCD using beam-splitters to save cost. Beam energy is measured with pyroelectric detectors coupled to $16 \mathrm{bit}, 100 \mathrm{kS} / \mathrm{s}$ digitizers on the FPGAs. Singlemode optical fiber is used to transmit pulse shape information to fast photodetectors (InGaAs, 1.2 $\mathrm{GHz}$ ) in the control room $15 \mathrm{~m}$ away. Backreflections from the target or misaligned optical surfaces are monitored with a combination of calorimeters and fiber-coupled photo-diodes at the polarizers of each Faraday isolator.

\section{Papers published in peer-reviewed journals}

- C. Niemann, W. Gekelman, C. Constantin, E. Everson, D. Schaeffer, S. Clark, D. Winske, A. Zylstra, P. Pribyl, S. Tripathi, D. Larson, S. Glenzer, A. Bondarenko Dynamics of exploding plasmas in a large magnetized plasma

Submitted to Phys. Plasmas (September 2012)

- A. Bondarenko, D. Schaeffer, E. Everson, C. Constantin, S. Clark, C. Niemann Feasibility of characterizing laser-ablated carbon plasmas via planar laser induced fluorescence

Rev. Sci. Instum. 83, 10E515 (2012)

- S. Clark, D. Schaeffer, A. Bondarenko, E. Everson, C. Constantin, C. Niemann Magnetic field measurements in low density plasmas using paramagnetic Faraday rotator glass

Rev. Sci. Instrum. 83, 10D503 (2012)

- D. Schaeffer, E. Everson, D. Winske, C. Constantin, A. Bondarenko, L. Morton, K. Flippo, D. Montgomery, S. Gaillard, C. Niemann

Generation of magnetized collisionless shocks by a novel, laser-driven magnetic piston

Physics of Plasma 19, 070702 (2012)

- C. Niemann, C. Constantin, D. Schaeffer, A. Tauschwitz, T. Weiland, Z. Lucky, W. Gekelman, E. Everson, D. Winske

High-energy Nd:glass laser facility for collisionless laboratory astrophysics JINST 7, P03010 (2012)

- G. Gregori, A. Ravasio, C. Murphy, K. Schaar, A. Baird, A. Bell, A. BenuzziMounaix, R. Bingham, C. Constantin, R. Drake, M. Edwards, E. Everson, C. Gregory, Y. Kuramitsu, W. Lau, J. Mithen, C. Niemann, H.S. Park, B. 
Remington, B. Reville, A. Robinson, D. Ryutov, Y. Sakawa, S. Yang, N.

Woolsey, M. Koenig, F. Miniati

Generation of scaled protogalactic seed magnetic fields in laser-produced shock waves

Nature 481, 480 (2012)

- D. Schaeffer, D. Montgomery, A. Bondarenko, L. Morton, R. Johnson, T. Shimada, C. Constantin, E. Everson, S. Letzring, S. Gaillard, K. Flippo, S. Glenzer, C. Niemann

Thomson Scattering Measurements of Temperature and Density in a LowDensity, Laser-Driven Magnetized Plasma

JINST 7, P02002 (2012)

- C. Niemann, A. Bondarenko, C. Constantin, E. Everson, K. Flippo, S. Gaillard, R. Johnson, S. Letzring, D. Montgomery, L. Morton, D. Schaeffer, T. Shimada, D. Winske,

Collisionless shocks in a large magnetized laser plasma plume

IEEE Trans. Plasma Science. 39(11), 2406 (2011)

- D. Schaeffer, N. Kugland, C. Constantin, E. Everson, B. VanCompernolle, C. Ebbers, S. Glenzer, C. Niemann

A scalable multipass laser cavity based on injection by frequency conversion for noncollective Thomson scattering

Rev. Sci. Instrum. 81, 10D518 (2010)

- A. Zylstra, C. Constantin, E. Everson, D. Schaeffer, N. Kugland, P. Pribyl, C. Niemann

Ion velocity distribution measurements in a magnetized laser plasma expansion

JINST 5, P06004 (2010)

- E. Everson, P. Pribyl, C. Constantin, A. Zylstra, D. Schaeffer, N. Kugland, C. Niemann

Design, construction, and calibration of a three-axis, high-frequency magnetic probe (B-dot probe) as a diagnostic for exploding plasmas Rev. Sci. Instrum. 80, 113505 (2009)

- C. Constantin, W. Gekelman, P. Pribyl, E. Everson, D. Schaeffer, N. Kugland, R. Presura, S. Neff, C. Plechaty, S. Vincena, A. Collette, S. Tripathi, M. Villagran Muniz, C. Niemann

Collisionless interaction of an energetic laser produced plasma with a large magnetoplasma

Astrophys. Space Sci. 322, 155 (2009) 